\title{
Surfaces of Strongly Confined Polymer Thin Films Studied by X-Ray Scattering
}

\author{
J. Wang \\ Experimental Facilities Division, Argonne National Laboratory, 9700 South Cass Avenue, Argonne, Illinois 60439 \\ M. Tolan \\ Institut für Experimentelle und Angewandte Physik der Universität Kiel, Leibnizstraße 19, 24098 Kiel, Germany \\ O. H. Seeck and S. K. Sinha \\ Experimental Facilities Division, Argonne National Laboratory, 9700 South Cass Avenue, Argonne, Illinois 60439 \\ O. Bahr, M. H. Rafailovich, and J. Sokolov \\ Department of Materials Science and Engineering, State University of New York at Stony Brook, Stony Brook, \\ New York 11794-2275 \\ (Received 19 October 1998)
}

\begin{abstract}
The surfaces of thermally annealed thin polystyrene films on silicon were investigated by specular and diffuse x-ray scattering. The data cannot be interpreted satisfactorily with capillary wave calculations assuming attractive van der Waals substrate film interactions. The analysis shows that polystyrene films coated on silicon substrates are in a strongly confined state even for film thicknesses much larger than the radius of gyration, possibly due to strong confinement that arises from the interaction at the polymer/substrate interface and the viscoelastic behavior of the thin films.
\end{abstract}

PACS numbers: 61.41.+e, 61.10.-i, 68.55.Jk

The field of confined liquids and, in particular, that of confined polymers have developed rapidly in the last ten years. Confinement complicates the physics of such films and may alter their properties drastically (see, e.g., [1-3]). X-ray scattering has proven to be one of the well-suited techniques for obtaining quantitative information about such interfaces. Whereas bulk liquids have been investigated rather extensively (see, e.g., [4$8]$ ), there are only a handful of quantitative investigations on fluid thin films [9-15], mainly due to difficulties in preparing samples with well-controlled thickness and interfacial environment. In this Letter, we present a systematic study, by $x$-ray specular and diffuse scattering measurements, of the surfaces of thermally annealed thin polymer films. To our surprise, we found that these thermally annealed polymer films are in a strongly confined state. They do not exhibit a liguidlike behavior.

We consider a thermally annealed and quenched thin polymer film on a solid substrate. As a generalization of results by Fredrickson et al. (see the Appendix of Ref. [16]), the excess free energy per oscillation mode $\Delta f$ of a distorted elastic film in lowest order perturbation theory is given by

$$
\Delta f=(1 / 2) \gamma_{\mathrm{eff}}\left(q^{2}+q_{0}^{2}\right)+\mu_{0} q+(3 / 2) \mu_{0} d^{-3} q^{-2},
$$

where $q$ is a lateral wave vector, $d$ denotes the thickness and $\mu_{0}$ the bulk shear modulus of the viscoelastic polymer, and the cutoff $q_{0}$ was introduced due to longrange subtrate/film interactions. The quantity $\gamma_{\mathrm{eff}}$ is an effective surface tension, which corresponds to the usual surface tension $\gamma$ in the case of a free liquid or polymer without confinement. In the case of van der Waals interactions with the substrate, the cutoff $q_{0}$ is given by $q_{0}=a / d^{2}$ with a length $a=\sqrt{A_{\text {eff }} / 2 \pi \gamma_{\text {eff }}}$, where the effective Hamaker constant $A_{\text {eff }}$ accounts for the interaction [10,17]. Equation (1) may be discussed in certain limits: In the case $\mu_{0}=0$ and $\gamma_{\text {eff }}=\gamma$, Eq. (1) describes a free liquid surface exhibiting capillary waves without viscoelasticity and confinement. The propagation of such capillary waves is hindered only by the attractive van der Waals interaction and by gravitation. The last two terms on the right-hand side of Eq. (1) consider the viscoelastic response of a thin film [16]. Here, $\mu_{0}$ is only a parameter accounting for elasticity which cannot be calculated within the framework of Ref. [16], since our system is not a thin film of molten polymer brushes. We note that, for large wave numbers $q$ and small $\mu_{0}$, the quadratic term dominates in Eq. (1). This term leads to particular power-law signatures in the $\mathrm{x}$-ray scattering data (see below).

To investigate liquid film surfaces, polystyrene (PS) with a molecular weight $M_{W}=90 \times 10^{3}$ and a radius of gyration $R_{G} \approx 80 \AA$ was used. The films were spun cast onto polished silicon wafers on which the native oxide was removed by dilute hydrofluoric acid solution. The film thickness ranges from 35 to $1676 \AA$ measured by ellipsometry and $\mathrm{x}$-ray reflectivity. The samples were annealed for approximately $12 \mathrm{~h}$ at $150{ }^{\circ} \mathrm{C}$ (well above the glass transition temperature $\sim 100{ }^{\circ} \mathrm{C}$ ) in high vacuum to reach thermal equilibrium conditions ( $p \sim 10^{-6} \mathrm{mbar}$, for preventing film dewetting). Upon removal from the vacuum oven, they were quenched to room temperature to a state of a "frozen liquid." Note that the thickest film 
is much thicker than $R_{G}$, hence the surface of this film is expected to behave like that of a free liquid. Atomic force microscopy images of the thin film surfaces were made twice on each sample to confirm that no dewetting has occurred during either the annealing process or the $\mathrm{x}$-ray measurements. The x-ray scattering experiments were performed at beam lines X10A and B of the National Synchrotron Light Source (NSLS) at Brookhaven National Laboratory. With the scattering geometry shown in the inset of Fig. 1 [scattering vector $\mathbf{q}=\mathbf{k}_{f}-\mathbf{k}_{i}=$ $\left(q_{x}, 0, q_{z}\right)$ ], a monochromatic beam (wavelength of $\lambda=$
$1.19 \AA$ A) was used. To access in-plane scattering vectors, $q_{x}$, the detector remained fixed while the incident angle was varied [18]. For each sample, the reflectivity and diffuse scattering at $q_{z}=0.15,0.20,0.30$, and $0.35 \AA^{-1}$ were measured. The samples were translated perpendicular to the beam by 2 to $3 \mathrm{~mm}$ every $20 \mathrm{~min}$ to avoid radiation damage to the polymer films.

Under the above experimental conditions, the scattering function $S(\mathbf{q})$, which is proportional to the observed intensity at point $\left(q_{x}, q_{z}\right)$ in reciprocal space, can be expressed as [5,18]

$$
S\left(q_{x}, q_{z}\right)=\frac{\Delta \rho^{2}}{q_{z}^{2}} \exp \left(-q_{z}^{2} \sigma^{2}\right) \int_{0}^{\infty} \exp \left\{q_{z}^{2} C(X)\right\} \exp \left(-\delta_{q_{x}}^{2} X^{2}\right) \cos \left(q_{x} X\right) d X
$$

where $C(X)$ is the diplacement-displacement correlation $C(R)$, defined as $\langle z(\mathbf{r}) z(\mathbf{r}+\mathbf{R})\rangle_{\mathbf{r}}$, averaged in the $y$ direction, with $z(\mathbf{r})$ being the displacement of the surface contour relative to some arbitrary origin at the lateral position r. The Fourier transform of this correlation function is essentially given by the inverse of the righthand side of Eq. (1) if the equipartition theorem is used [6]. For this purpose, the expression given by Eq. (1) is sufficient and the mode amplitudes are not necessary in the further calculations. $\sigma$ in Eq. (2) is the rms roughness and $\Delta \rho$ is the electron density contrast at the polymer/air interface. Wide-open slits in the $y$ direction lead to an $q_{x}^{\eta-1}$ power-law decay of the diffuse scattering instead of the usual $q_{x}^{\eta-2}$ decay for the nonintegrated case where the quantity $\eta$ will be specified below (for further details, see Ref. [5]). The narrow slits in the scattering plane were

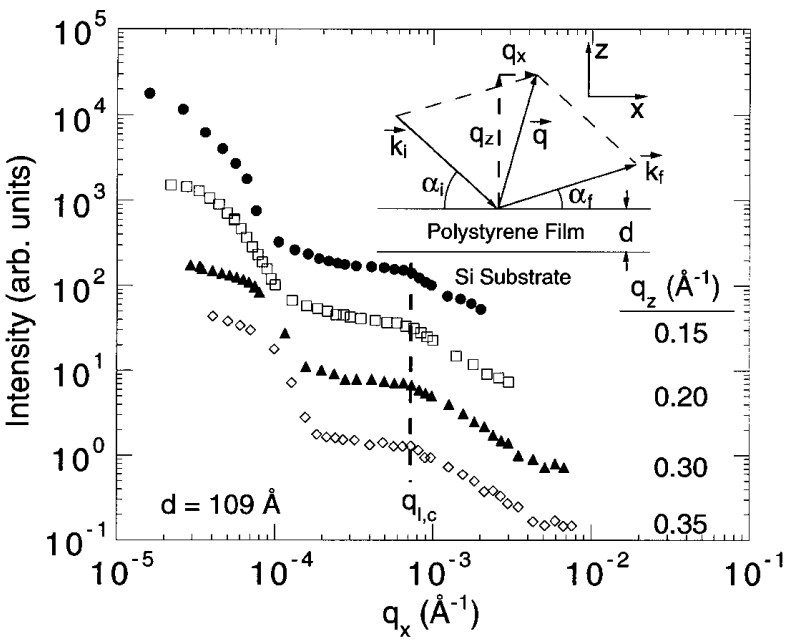

FIG. 1. Transverse scans along $q_{x}$ at different $q_{z}$ values for a polystyrene film of thickness $d=109 \AA$ on silicon. The vertical broken line indicates the location of the lower cutoff $q_{l, c}$ and shows that this location is $q_{z}$ independent. The inset shows the in-plane scattering geometry: The wave vectors of the incident and scattered $\mathrm{x}$ rays are $\mathbf{k}_{i}$ and $\mathbf{k}_{f}$, with the incidence and exit angle $\alpha_{i}$ and $\alpha_{f}$, respectively. The momentum transfer is defined by $\mathbf{q}=\mathbf{k}_{f}-\mathbf{k}_{i}=\left(q_{x}, q_{z}\right)$. taken into account by the real-space resolution function $\exp \left(-\delta_{q_{x}}^{2} R^{2}\right)$. In the present study, diffuse scattering from the substrate/polymer interface was neglected because the substrate was very smooth $(\sigma<3 \AA)$, resulting in its diffuse scattering intensity being 2 orders of magnitude lower than that of the PS films.

Figures 1 and 2 depict transverse diffuse scans for the series of PS samples. The following form was used as the correlation function in Eq. (2) for the fits: $C(R)=$ $(B / 2) K_{0}\left(q_{l, c} \sqrt{R^{2}+r_{0}^{2}}\right)$ with the modified Bessel function $K_{0}(X)$ and two adjustable parameters $B$ and $q_{l, c}$. A low-length cutoff $r_{0}$ was introduced, which is related to

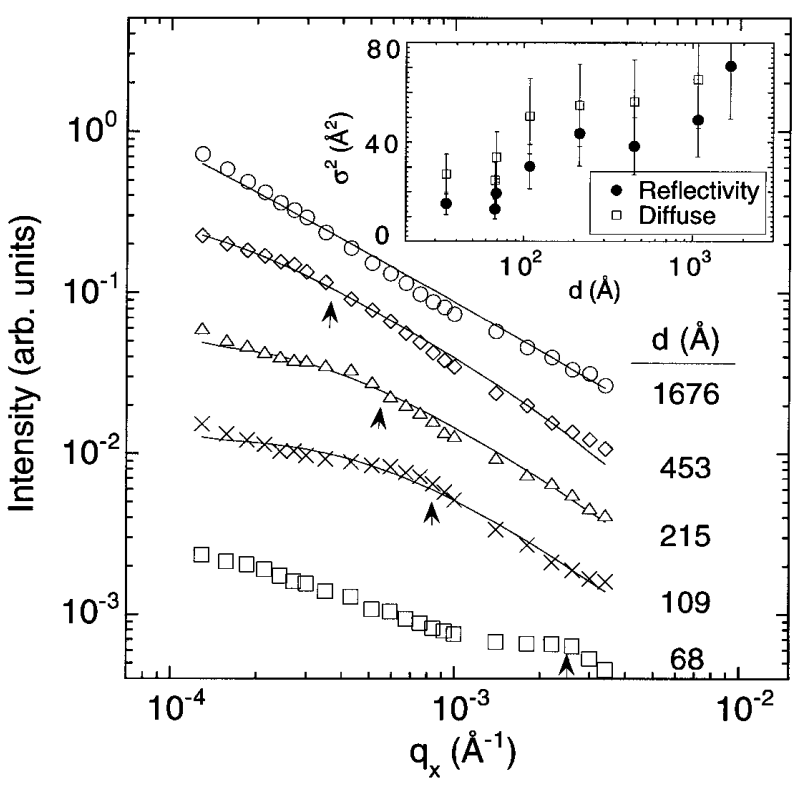

FIG. 2. Diffuse scattering from polystyrene films on silicon substrates with film thicknesses $d=35, \ldots, 1676 \AA$. Transverse scans along $q_{x}$ for fixed $q_{z}=0.2 \AA^{-1}$ are shown. The lines are fits with a lower cutoff $q_{l, c}$ (arrows) as a parameter (not shown for the $d=68 \AA$ film). For the thickest film, the cutoff is hidden under the specular peak, i.e., in the region $q_{x}<10^{-4} \AA^{-1}$. The inset depicts the mean-square roughness $\sigma^{2}$ vs $d$ as measured by specular reflectivity (circles) and calculated from the integrated diffuse scattering (squares). 
the finite size of the molecules and the bond lengths, and which defines an upper wave-number cutoff $q_{u, c}=$ $2 \pi / r_{0}$. This cutoff $[7,8]$ is on the order of $1-10 \AA^{-1}$ in our study [19]. We note that in the case of free capillary waves, $B=k_{B} T / \pi \gamma$ and $q_{l, c}$ would be identical to the van der Waals cutoff $q_{0}=a / d^{2}$ and the correlation function above would be exact $[5,6,10]$. It turns out that this correlation function may also be used in the limit of small $\mu_{0}$, which is relevant to our study [20].

The data for the series of polystyrene films show three major signatures that can be associated with particular terms of the free-energy expression given by Eq. (1):

(i) The mean-square roughness $\sigma^{2}$ (see inset of Fig. 2) of the film surfaces, as obtained by fitting the specular reflectivity data, increases logarithmically with increasing film thickness (for clarity $\sigma^{2}$ is presented on a linear scale). An independent method, namely calculating $\sigma^{2}$ from the diffuse scattering curves according to Eq. (2), confirmed those values.

(ii) The "kink" in the curves of Figs. 1 and 2 indicates the location of the cutoff $q_{l, c}$ which was a fit parameter. As shown in Fig. 3, $q_{l, c}$ may be approximated by the relationship $q_{l, c}=b / d^{m}$ with two parameters $b$ and $m$ that will be specified below. In the transverse diffuse scans, this cutoff separates a power law from a rather flat region.

(iii) From the data shown in Figs. 1 and 2, we found that the power law part, i.e., the scattering for $q_{x}>q_{l, c}$, may be approximated by $I\left(q_{x}\right) \sim q_{x}^{\eta-1}$ with $\eta \approx 0$.

The conclusions that can be drawn from these three basic properties will now be discussed: A logarithmic increase of the rms roughness with the film thickness would be expected for capillary waves on thin liquid films. Recently, this has been confirmed for polymer/ polymer interfaces by neutron reflectivity measurements [21]. However, the error bars of the data shown in the

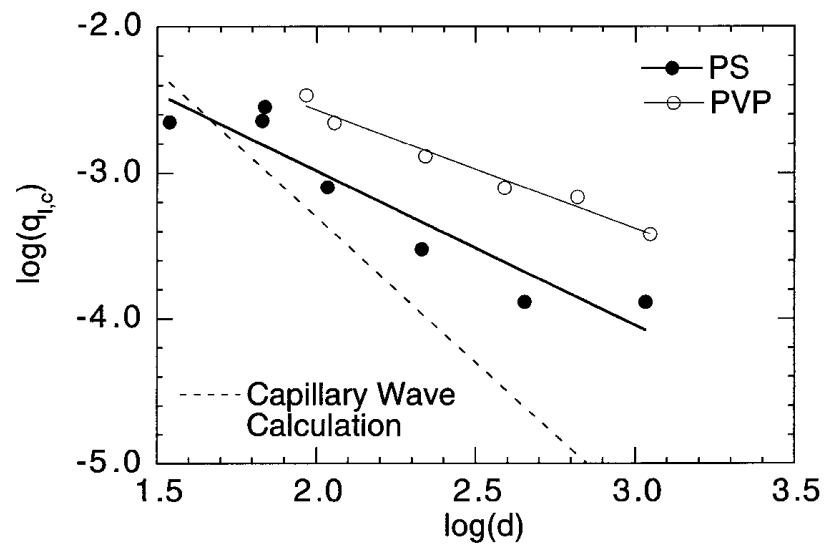

FIG. 3. Log-log plot of the low wave-number cutoff $q_{l, c}$ as a function of the film thickness for the series of PS samples (solid circles) and a PVP series (open circles). The solid lines are power-law fits according to $q_{l, c}=b / d^{m}$ yielding $m=1.0$ and $m=0.8$ for PS and PVP, respectively. The dashed line corresponds to the case of free capillary waves with $m=2$ and $b=5 \AA$. inset of Fig. 2 are too large to confirm unambiguously a logarithmic dependence of $\sigma^{2}$ on $d$. Moreover, it can be proven analytically that this logarithmic increase is a general feature caused only by the leading $q^{2}$ term of the free-energy expression [see Eq. (1)]. The absolute values, however, are also sensitive to the other terms of Eq. (1).

The second signature, the thickness-dependent location of the cutoff, is highlighted in Fig. 3. Here pure van der Waals interactions without viscoelasticity, i.e., assuming $\mu_{0}=0$ in Eq. (1), would predict a relationship $q_{l, c}=$ $a / d^{2}$, corresponding to $m=2$ and $b=a$ with $a \sim 5 \AA$ (see the dashed line in Fig. 3) in the above-mentioned case (ii). However, Fig. 3 reveals that our data suggest $m=1.0 \pm 0.2$ instead of $m=2$. Included also in this figure are the results of a preliminary measurement of a similar series of polyvinyl-pyridine (PVP) films where we found $m \approx 0.8$, a value similar to the PS result and also far way from the capillary waves prediction. It is known that PVP reacts more strongly with a silicon surface, so that the viscoelastic nature of the thin films becomes more pronounced. This may be understood in a more quantitative manner if we assume a finite shear modulus $\mu_{0}$, i.e., a highly viscous liquid, in Eq. (1). Neglecting van der Waals forces $\left[q_{0} \approx 0\right.$ in Eq. (1)], it is simple to show that the location of the cutoff scales as $q_{l, c} \sim 1 / d^{0.75 \ldots 1}[16,22]$. The location of this cutoff is determined by the second and third terms on the righthand side of Eq. (1). These terms are the high and low $q$ contributions of elastic distortions to the wave-number spectrum (see the Appendix of Ref. [16]). Since we found $m \approx 1$ for PS and $m \approx 0.8$ for PVP, our data favors the model of viscoelasticity over that of free capillary waves.

The last issue, the low $\eta$ value, is more subtle. It can be shown that from a free-energy expression such as Eq. (1), a power law follows for $S\left(q_{x}, q_{z}\right)$ and $q_{x}>q_{l, c}$ [5]. One gets $S\left(q_{x}, q_{z}\right) \sim q_{x}^{\eta-1}$ with $\eta=B q_{z}^{2} / 2$ and $B=k_{B} T / \pi \gamma_{\mathrm{eff}}$. The source for this particular power law is again the leading quadratic $q$ term on the righthand side of Eq. (1), which describes surface undulations on lateral length scales smaller than $R_{G}$. While we found a value very close to zero for all $q_{z}$ values and all film thicknesses (even for the $1676 \AA$ thick film), one would expect, e.g., $\eta \approx 0.15$ for $q_{z}=0.20 \AA^{-1}$ as calculated from a $B$ value of $7.4 \AA^{2}$ obtained with the bulk-surface tension of PS. The very low $\eta$ and thus high surface tension may be caused by corrections to the $q^{2}$ term of the free energy. For example, curvature corrections as discussed by Napiórkowski and Dietrich [23,24] or modifications of the simple $1 / r^{6}$ pair potential [25], which are very likely for polymers, would yield considerably smaller $\eta$ values since they effectively can be described by an enhanced surface tension $\gamma_{\text {eff }}$. But the larger $\gamma_{\text {eff }}$ would have yielded roughness smaller than that obtained from the reflectivity and diffuse scattering measurements [5].

PS and PVP stick on Si surfaces. Thus one may suspect that the unusual nonliquidlike behavior of the films 
is caused by the strong confinement, which is induced by the substrate interaction. There are other arguments in favor of this explanation. It was shown recently [15] that polymers that do not interact with the substrate indeed show capillary waves on their surfaces. Furthermore, we monitored in situ with diffuse x-ray scattering measurements that the cutoff appears only after annealing the films as explained above. Hence the interaction with the substrate has to be thermally activated. Preliminary experiments have shown that the interaction may be screened by a thin polymer layer underneath the PS film or may be totally avoided in the case of free-standing films. Thus, one may identify the strong interaction and hence strong confinement at the $\mathrm{Si}\left(\right.$ or $\left.\mathrm{SiO}_{x}\right)$ /polymer interface as the source for the viscoelastic behavior and unusual high surface tension that we found for the thin PS and PVP films in our study.

In summary, we have shown, for the first time, that long wavelength fluctuations are strongly suppressed due to the interactions between the thin film and the substrate, which transforms the film into a very viscous gel-type state. Such suppression results in a low wave-number cutoff varying as a function of the film thickness, which can be clearly identified by $x$-ray diffuse scattering measurements. We found that the substrate-induced confinement is present even for films with thicknesses many times greater than the radius of gyration. We also attempt to explain the experimental results quantitatively with a theory essentially based on viscoelasticity. The experiments presented in this Letter, as well as future studies, should shed light on a complete understanding of the dynamics at the surfaces of thin liquid and glassy films, interactions between polymer films and substrates, and viscoelastic properties of polymeric material under confinement.

We thank the Exxon beamline staff at NSLS for technical assistance, and A. K. Sood, K. W. Shin, X. Pu, and A. Gibaud for discussion. NSLS is supported by the U.S. Department of Energy, Division of Materials Sciences and Division of Chemical Sciences. This work was partially supported by DOE under BES, Contract No. W-31-109-ENG-38.

[1] Polymers in Confined Environments, edited by S. Granick, Advances in Polymer Science (Springer, New York, 1998), Vol. 138.

[2] W.E. Wallace, J.H. van Zanten, and W.L. Wu, Phys. Rev. E 52, R3329 (1995).

[3] J.H. van Zanten, W.E. Wallace, and W.L. Wu, Phys. Rev. E 53, R2053 (1996).

[4] A. Braslau, M. Deutsch, P. S. Pershan, A. H. Weiss, J. AlsNielsen, and J. Bohr, Phys. Rev. Lett. 54, 114 (1985).
[5] M. K. Sanyal, S. K. Sinha, K. G. Huang, and B. M. Ocko, Phys. Rev. Lett. 66, 628 (1991).

[6] J. Daillant, L. Bosio, B. Harzallah, and J. J. Benatter, J. Phys. II (France) 1, 149 (1991).

[7] B. M. Ocko, X.Z. Wu, E. B. Sirota, S. K. Sinha, and M. Deutsch, Phys. Rev. Lett. 72, 242 (1994).

[8] O. M. Magnussen, B. M. Ocko, M. J. Regan, K. Penanen, P. S. Pershan, and M. Deutsch, Phys. Rev. Lett. 74, 4444 (1995).

[9] S. Garoff, E. B. Sirota, S. K. Sinha, and H. B. Stanley, J. Chem. Phys. 90, 7505 (1989).

[10] I. M. Tidswell, T.A. Rabedeau, P.S. Pershan, and S.D. Kosowsky, Phys. Rev. Lett. 66, 2108 (1991).

[11] L. B. Lurio, T. A. Rabedeau, P.S. Pershan, I. F. Silvera, M. Deutsch, S. D. Kosowsky, and B. M. Ocko, Phys. Rev. Lett. 68, 2628 (1992).

[12] J. Daillant and O. Bélorgey, J. Chem. Phys. 97, 5824 (1992).

[13] E. A. L. Mol, G. C. L. Wong, J.-M. Petit, F. Rieutord, and W. H. de Jeu, Phys. Rev. Lett. 79, 3439 (1997).

[14] A. Plech, U. Klemradt, H. Metzger, and J. Peisl, J. Phys. Condens. Matter 10, 971 (1998).

[15] M. Tolan, O. H. Seeck, J.-P. Schlomka, W. Press, J. Wang, S. K. Sinha, Z. Li, M.H. Rafailovich, and J. Sokolov, Phys. Rev. Lett. 81, 2731 (1998).

[16] G. H. Fredrickson, A. Ajdari, L. Leibler, and J.-P. Carton, Macromolecules 25, 2882 (1992).

[17] J. Israelachvili, Intramolecular Surface Forces (Academic Press, New York, 1992), 2nd ed.

[18] S. K. Sinha, E. B. Sirota, S. Garoff, and H. B. Stanley, Phys. Rev. B 38, 2297 (1988).

[19] From the fits of the diffuse scattering data, we have estimated $r_{0}$ to be $7 \AA$. We are not able to obtain the exact $r_{0}$ value due to the low scattering intensity at large $q_{x}$ and the simultaneous increasing of the influence of the so-called "Yoneda peak" caused by dynamical scattering effects.

[20] To be exact, instead of $K_{0}(x)$, Eq. (1) yields $C(R)=$ $(B / 2) \operatorname{ker}\left(q_{l, c} \sqrt{R^{2}+r_{0}^{2}}\right)$ with a Kelvin function $\operatorname{ker}(X)$, in which $B, q_{l, c}$, and $r_{0}$ have the same definition as in $C(R)$ based on $K_{0}(X)$. This Kelvin function has the same asymptotic behavior as $K_{0}(X)$ and yields the same fit results for the three parameters.

[21] M. Sferrazza, C. Xiao, R. A. L. Jones, D. G. Bucknall, J. Webster, and J. Penfold, Phys. Rev. Lett. 78, 3693 (1997).

[22] We note here that the explicit discussion in Ref. [16] would yield either a $q_{l, c} \sim 1 / d$ or a $q_{l, c} \sim 1 / d^{0.75}$ relationship with a transition regime depending on the film thickness. However, since we cover only 1.5 orders of magnitude with our film thicknesses and the error bars of $q_{l, c}$ are rather large, this transition between the two power laws cannot be detected.

[23] M. Napiórkowski and S. Dietrich, Phys. Rev. E 47, 1836 (1993).

[24] M. Napiórkowski and S. Dietrich, Z. Phys. B 97, 511 (1995).

[25] S. Dietrich, J. Phys. Condens. Matter 8, 9127 (1996). 